\title{
The Research of Bad Review on the Evaluation of the Network's Influence on Consumer Choice
}

\author{
ZHU Donglian, LI yali \\ College of Finance and Economics ,Jiangxi University of Technology ,Nanchang,China, 330098
}

Keywords: Online evaluation; Poor evaluation; Consumers' choice; Taobao

\begin{abstract}
Ever since the 21st century, with the rapid development and popularization of the Internet in China, which has changed people's way of life, new business mode and economic behavior also arise at the historic moment. In the traditional way of shopping, people share about consumption experience of commodities or services through face-to-face communication, which is online evaluation. Online evaluation affects consumers' purchase decisions through affecting their trust, so this paper proposes the research of online evaluation's influence on consumers' choice based on this research background.
\end{abstract}

\section{Research and Implementation of Empirical Design}

Data Collection and Organization. Data Collection:This research collected data through questionnaires, so it can more effectively identify and verify online shoppers to let consumers fill in online questionnaires under similar environment. Besides, online surveys are more in line with the interests and hobbies of the Internet users and more likely to receive positive response from the respondents, who are more likely to be fill in the questionnaire more seriously. In this survey, questionnaires are distributed online, that is, to distribute survey questionnaires through professional questionnaire survey network (questionnaire star). A total of 105 questionnaires are collected in this research and 105 of them are valid questionnaires.

Data Analysis:

This research shows that among today's online shopping population, there are significantly more females than males, among which $63.81 \%$ of them are aged between 18 and 24 with a vast majority of $77.14 \%$ having bachelor's degree. All of the respondents in the survey have some experience that their online shopping choices are influenced by online evaluation. While shopping online, 94.29\% consumers will generally choose to browse the online evaluation of this commodity, and $81.9 \%$ respondents say that commodity with a large amount of online evaluation will attract their attention, which indicates that online evaluation has some influence on consumption choice.

In terms of the quality of commodity's online evaluation, $72.38 \%$ of people consent to the item "these evaluations have high quality"; only $63.81 \%$ of people agree or quietly agree with that "these evaluations leave me a deep impression"; only $62.86 \%$ of people think that "these evaluations are convincing"; $67.62 \%$ of people agree that "these evaluations are very substantial" and only $63.81 \%$ of people recognize that "these evaluations are made with a serious attitude". Overall, respondents have a generally low recognition of the quality of online evaluation, and data show that consumers believe that these evaluations are not so convincing and do not reflect so serious attitude. 
In terms of the emotional tendency part of customers' online evaluation, only $30.48 \%$ of respondents believe "these online evaluations are positive evaluations", showing that customers do not have enough trust in these evaluations.

With regard to online evaluation's influence on your purchase decision, these online evaluations provide great help for me to make the purchase choice as well as some different opinions about the product.

In terms of what kind of poor evaluation will affect your purchase decisions, nearly all the people choose evaluation on the shop and product quality, showing that customers are most focused on the shop's evaluation and the product's quality, which have the biggest impact on customers' purchase decisions. $56.6 \%$ of respondents choose the after-sale service, indicating that customers are still very focused on the product's follow-up service. $52.83 \%$ of respondents choose customer service because good customer service can attract customers to buy the commodity.

\section{Research Conclusion}

This paper proves the theoretical relationship between online evaluation and consumers' choice through collecting and analyzing data, and gets the following research conclusion while combing the actual situation of Taobao:

1) It has been found that the spread of negative online evaluation not only affects customers' online shopping, but also their off-line shopping. Besides, it can be known from previous research conclusions that negative evaluations have greater influence than positive evaluations. In the Internet environment, with fast spreading speed, profound influence and wide range of audiences, negative evaluation influences customers' purchase intention to a greater extent.

2) Customers' online evaluation has great influence on customers' attention and choice of commodities. With the constant popularization of the Internet and people's increasingly deepening understanding of online purchase, more and more customers conduct online shopping on the third party website like Taobao. It can be found by subdividing these online customers that in addition to those college students who are already keen on online shopping, many Internet users of different ages, positions and identities also join the group of online customers, and the constitution of customers are developing to a diversification direction. However, the decision-making process and index system for reference before they make the purchase decisions are convergent.

3) The basis of making a choice is the trust and recognition of online shopping value. It can be found in the research that customers hope to get the commodities same as the description, and the commodity's price is an important basis of reference for customers in shopping. To sum up, only after comparing the online and offline similar products and the online products have a large price advantage, customers will choose to buy them online. This show that customers choose to buy the products online not just blindly following the trend or in the pursuit of fashion, but to pursue the maximum value of products from an economic perspective.

4)Experience exchanges between customers will greatly affect customers' trust in the commodity, even their purchase decision. It is found through research that if a customer has successful purchase experience at a certain online shop, then when he wants to buy the similar commodity, he would probably choose this shop and share this successful shopping experience with relatives, friends or strangers who want to buy similar products. This information spread by relatives and friends is easy to cause resonance and get recognition. 
5) It can be found through researches that customers are not entirely believing in online evaluation, but once this trust is establish, it will last for longer. During the process of online shopping, customers can have a brief recognition of a shop or commodity, but it is not easy to establish confidence in evaluations about this commodity. Only after having successful shopping experience and a deeper understanding of the commodity and service, can customers establish confidence.

\section{Marketing Implications}

Master Negative Online Evaluation and Make Timely Remedy. Through research we know that no matter the evaluation is of high quality or low quality, once it is negative evaluation, it will produce some negative influence on customers' purchase decisions to some extent. Therefore, enterprises should pay attention to the management of online evaluations. While browsing various evaluations in online shopping, we can find that some sellers can response to negative evaluations timely, no matter apology or explanation. However, not so many online sellers response to negative evaluations and a few of them response to all the negative evaluations. Therefore, it is suggested that enterprises should invest resources, establish targeted department and personnel, include online evaluation management into the enterprise strategic marketing and integrate the marketing spreading ideas for management and operation.

Pay Attention to the Quality of Online Evaluation and Reduce Negative Influence. We can see from the research results that negative evaluations of different qualities have different influence on customers: the higher the quality, the greater the influence is, thus it is more likely to change customers' purchase decision. Sellers should encourage customers to publish some comprehensive, true, fair and objective positive information about the commodity or service. The more high-quality online evaluation will have greater impact on customers' choices, so online sellers can adopt some preferential ways like discount, giving gifts and accumulative integrals to guide and stimulate customers to publish some high-quality and positive evaluations about the commodity so as to improve the customers' trust and promote their purchase decision. While encountering negative online evaluations about the commodity or service, sellers should pay attention to them and analyze them immediately and publish high-quality positive evaluation timely so as to reduce the negative influence of these negative evaluations.

Sellers Should Actively Reduce the Amount of Medium and Poor Evaluation. It is easy for online evaluations to be copied and shared by customers, and enterprises' potential customers are very likely to contact these similar negative online evaluations about the commodity. It is found in research that poor online evaluation can significantly affect customers' purchase intention. The more negative online evaluations there are, the greater degree that customers' purchase decisions are affected. Therefore, sellers should make timely remedy for negative online evaluations, trying to minimize the amount of negative online evaluation so as to reduce their impact on customers' purchase decisions.

Enterprises Should Strengthen the Management of High-quality and Professional Evaluation Publishers. Enterprises can actively seek online evaluation marketers and form good interaction and exchange with them so as to reduce the probability of negative online evaluation. Meanwhile, they can invite these marketing experts to actively participate in their marketing activities so as to leave a good impression on them and let them spread positive information about the commodity to the market through online evaluation. 


\section{Conclusion}

E-commerce in China has already entered the era of rapid development, and online shopping is becoming more and more popular with an increasing amount of research about e-commerce. Therefore, it is very necessary to research the influence of online medium and poor evaluation's influence on customers' choice.

It can be found through research that the spread of negative online evaluation can affect not only customers' online shopping, but also their offline shopping. Besides, it can be known from previous research conclusions that negative evaluation has greater impact on positive evaluation. Currently, domestic researches about online evaluation's impact on customers' choice are taking the Internet as the object, but online evaluations contain a wide range of contents without strong pertinence.

The research in this paper about medium and poor online evaluations' impact on customers' choice is more targeted with great reference value for future researches. In addition, the conclusion obtained through research provides great guidance and help to the future operation and development of online enterprises.

\section{References}

[1] Tianyu. Online Evaluation's Influence on Consumers' Purchase Intentions. Beijing University of Post and Telecommunications, 2013(3).

[2] Zhao Ying, Cui Jin. Shopping Evaluation's Influence on Online Consumption. Scientific Information, 2010.

[3] Li Huiying. Research of Online Evaluation's Influence on Consumers' Perception and Enterprise Commodity Sales, Harbin Industrial University, 2013.

[4] Zhang Xiaodong, Zhu Min. "Research and Discussion of Online Evaluation's Influence on Consumers' Purchase Behaviors', Consumption Economics, 03 issue of 2011.

[5] Peng Lan, Zhou Qihai, Qiu Jiangtao. Research of Consumers' Online Evaluation's Helpful Influencing Factor Model, Computer Science, 08 issue of 2011.

[6] Li Xin. Research of Online Evaluation and Perception Value's Influence on Consumrs' Purchase Intention, Henan Social Science, 03 issue of 2010.

[7] Robert P A. Merino M C. Consumer information search behavior and the internet[J]. Psychology \& Marketing, $2013>$ 20(2): 99-121

[8] Chen P Y,Wu s Y? J Yoou. The impact d online recommendations and consumer feedback on sales[A].//: Chris V Z,Steve P(Eds). Proceedings of the International Conference On Information J. AIS Electronic Library, WashingtonDC.2011:711-724

[9] Chevfier J A,Mayzlin D. The effect of word-Of-mouth on sales: Onfine bookreviews. Journal of Marketing Research, 2011,43(3): 345-354

[10]Dellarocns C.Awad N.Zhang x. Exploring the value of online product reviews in forecasting sales: The case of motion pictures Journal of Interactive Marketing, 2012. 21(4):23-45 